\title{
Encalhe de peixe-boi-marinho-das-Antilhas (Trichechus manatus manatus Linnaeus, 1758) na praia de Panaquatira, Maranhão, Brasil
}

\author{
Fábio Pereira Conceição ${ }^{1 *}$, Anderson Campelo Costa ${ }^{2}$, Amanda Sousa $^{2}$, Rogério Bugarin ${ }^{2}$, \\ Laura Andrade-Reis ${ }^{3} \&$ Marcelo Derzi Vidal ${ }^{1}$
}

${ }^{1}$ Centro Nacional de Pesquisa e Conservação da Sociobiodiversidade Associada a Povos e Comunidades Tradicionais (CNPT/ICMBio), São Luís,
$M A$, Brasil.
${ }^{2}$ Universidade Federal do Maranhão, São Luís, MA, Brasil.
${ }^{3}$ Reserva Extrativista Marinha de Cururupu (REMC/ICMBio), São Luís, MA, Brasil.

*E-mail para correspondência: fabio_pconceicao@hotmail.com

\begin{abstract}
RESUMO
O peixe-boi-marinho-das-Antilhas (Trichechus manatus manatus) apresenta distribuição geográfica que se estende por todo o Caribe e Américas Central e do Sul. No Brasil, a espécie é considerada ameaçada de extinção devido, sobretudo, a fatores antrópicos, como emalhes em rede de pesca, perda de habitat devido à ocupação desordenada do litoral e uso inadequado das áreas marinhas e estuarinas. Neste artigo, relatamos o encalhe e morte de um espécime de peixe-boi-marinho-das-Antilhas no litoral maranhense. Por meio de procedimentos morfométricos, constatou-se ser um animal do sexo masculino medindo 3,14 m de comprimento por 2,30 m de circunferência em sua região corporal de maior largura. Em seu corpo não foi encontrado nenhum corte, perfuração ou marcas de emalhamento em redes de pesca, sugerindo inicialmente a não ocorrência de uma interação antrópica direta. A realização de inventários que possam estimar a abundância e a densidade de suas populações, bem como o monitoramento dos encalhes de animais vivos ou mortos são fundamentais para subsidiar ações de manejo e garantir a preservação da espécie no litoral maranhense.
\end{abstract}

Palavras-chave: Espécie ameaçada, Litoral brasileiro, Mamífero aquático

\section{ABSTRACT}

Record of a stranded Antillean manatee (Trichechus manatus manatus Linnaeus, 1758) at Panaquatira Beach, Maranhão, Brazil. The Antillean manatee (Trichechus manatus manatus Linnaeus, 1758) is distributed throughout the Caribbean, Central and South America. In Brazil, it is considered threatened of extinction mainly due to anthropogenic factors such as incidental captures in fishing net, habitat loss due to disordered occupation of the coast and inappropriate use of marine and estuarine areas. In this article we report the stranding of a specimen of Antillean manatee in the coast of Maranhão. Through morphometric procedures it was found to be a male animal measuring $3.14 \mathrm{~m}$ in length and $2.30 \mathrm{~m}$ in circumference in its widest body region. No injury, perforation or entanglement marks were found in his body, initially suggesting that no direct anthropic interaction occurred. The carrying out of inventories that can estimate the abundance and density of their populations, as well as the monitoring of stranding of live or dead animals are fundamental to support management actions and ensure the preservation of the species along the coast of Maranhão.

Keywords: Aquatic mammal, Brazilian coast, Endangered species 
O peixe-boi-marinho-das-Antilhas (Trichechus manatus manatus Linnaeus, 1758), mamífero aquático da ordem Sirenia, apresenta uma distribuição geográfica por todo o Caribe e Américas Central e do Sul (Hatt, 1934; Domning \& Hayek, 1986). No Brasil, estudos sugerem uma distribuição descontínua que vai desde o Amapá a Alagoas, em uma faixa de aproximadamente 5.000 quilômetros na costa (Luna et al., 2010; Lima et al., 2012).

Mamífero de grande porte, podendo atingir 4,5 m e pesar $600 \mathrm{~kg}$ quando adulto (Rosas \& Pimentel, 2001), o peixe-boi-marinho-das-Antilhas apresenta pele áspera, de coloração acinzentada e, diferentemente do peixe-boi amazônico, unhas são visíveis nas nadadeiras peitorais (Luna et al., 2018).

A dieta do peixe-boi-marinho-das-Antilhas é composta por diferentes tipos de algas e capins marinhos, mangue, aninga, paturá, mururé e junco (Best \& Teixeira, 1982; Paludo, 1997; Borges et al., 2008). Apesar de majoritariamente herbívoro, também já foram registrados consumo de invertebrados associados à vegetação e ingestão eventual de peixes, itens que podem representar uma importante fonte de proteína (Powell, 1978; Hartman, 1979; Borges et al., 2008).

O peixe-boi-marinho-das-Antilhas consta na lista brasileira de espécies ameaçadas de extinção (MMA, 2014) na categoria Em Perigo, sendo que as principais ameaças à espécie estão relacionadas a fatores antrópicos, como a perda de habitat devido à ocupação desordenada do litoral e o uso inadequado das áreas marinhas e estuarinas, o que implica em declínio na qualidade do habitat da espécie, mesmo em locais menos populosos (Luna et al., 2018). Este cenário fez com que o Instituto Chico Mendes de Conservação da Biodiversidade (ICMBio) criasse em 2018 o Plano de Ação de Nacional para Conservação dos Peixes-bois Marinhos, que tem como objetivo reduzir os efeitos das atividades antrópicas sobre as populações naturais da espécie, ampliar o conhecimento aplicado a sua conservação e aperfeiçoar as ações de conservação ex situ (MMA, 2018).

Em 2010 foi realizado um levantamento da distribuição do peixe-boi marinho na Costa Norte do Brasil. Nesse estudo constatou-se a ocorrência da espécie no Golfão Maranhense, principalmente nas localidades de Porto Grande e Igaraú, na porção leste da Ilha de São Luís, na Ilha dos Caranguejos e na Baía do Tubarão, nas localidades Axuí, Carrapatal, Manuma e Porto Rosa (Luna et al., 2010). Nesta região, as elevadas amplitudes de marés proporcionam vastas planícies fluviais com forte influência marinha, que somadas à existência de vegetação e abrigos junto à costa oferecem alimentação e proteção aos peixesboi (Luna et al., 2010).

No Maranhão, o Centro Nacional de Pesquisa e Conservação de Mamíferos Aquáticos (CMA/ICMBio) iniciou o monitoramento das populações do peixe-boi marinho em 2002, com a implantação do sítio de observação na Praia de Guarapiranga, município de São José de Ribamar. No ano seguinte, as atividades de monitoramento populacional foram expandidas para duas outras áreas de 
ocorrência da espécie - Ilha do Gato, município de Humberto de Campos, e Ponta de Pedras, município de Alcântara (Alvite et al., 2006; Alvite, 2008).

No entanto, em março de 2015, a Portaria ICMBio $\mathrm{n}^{0} 16$ determinou o fechamento da base avançada do CMA/ICMBio no Maranhão e, consequentemente, as atividades deixaram de ser desenvolvidas. Desde então, não se conhece a situação das populações de peixes-boi marinhos no litoral maranhense, ainda que as ações relacionadas a espécie tenham sido realocadas para o Centro Nacional de Pesquisa e Conservação da Biodiversidade Marinha do Nordeste (CEPENE/ICMBio) e diversas atividades integrantes do Plano de Ação de Nacional para Conservação dos Peixes-bois Marinhos venham sendo realizadas em outras áreas.

O presente trabalho relata o encalhe de um exemplar de peixe-boi-marinho-das-Antilhas no litoral maranhense e apresenta os procedimentos realizados por uma equipe de profissionais que vem atuando espontaneamente em situações que envolvem mamíferos aquáticos nesta região.

Em cinco de maio de 2019, a equipe foi contatada pelo Corpo de Bombeiros do Maranhão que informou ter sido avistado um peixe-boi marinho encalhado na praia de Panaquatira, município de São José de Ribamar. Segundo os bombeiros, o animal encalhou no dia anterior e a maré o teria levado para uma área de arrebentação.

Chegando ao local do encalhe (Figura 1) a equipe encontrou o exemplar já sem vida, em decúbito dorsal, em uma área de pedral próxima a muros de alvenaria que delimitam residências locais (Figura 2).

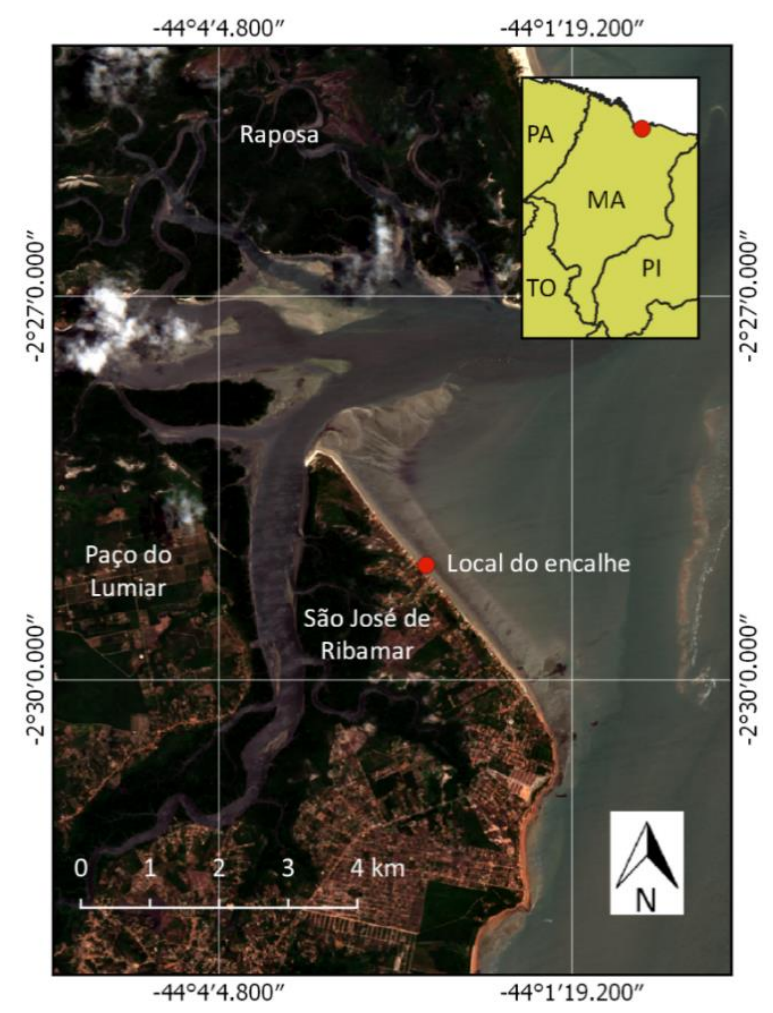

Figura 1. Mapa indicando a localização do encalhe do peixe-boi-marinho-das-Antilhas na praia de Panaquatira, São José de Ribamar - MA. 


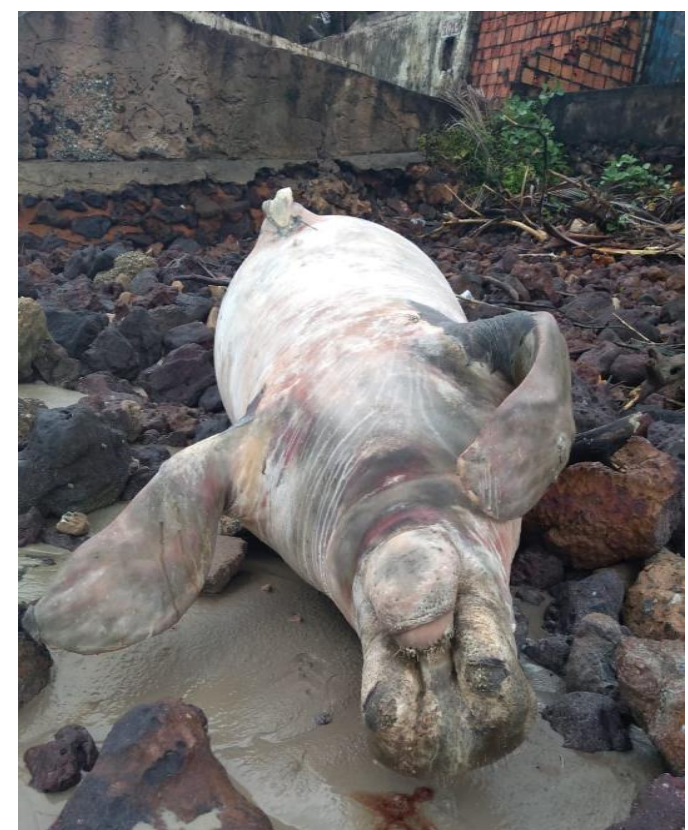

Figura 2. Exemplar de peixe-boi-marinho-das-Antilhas encontrado próximo a residências na praia de Panaquatira, São José de Ribamar - MA.

Após detalhada observação visual externa do corpo do animal, não foi encontrado nenhum corte, perfuração ou marcas de emalhamento em redes de pesca, sugerindo inicialmente a não ocorrência de uma interação antrópica direta. Por meio de procedimentos morfométricos (Figura 3) e observação da distância entre as regiões genital e anal (Figura 4), constatou-se ser o animal do sexo masculino, medindo 3,14 m de comprimento por 2,30 m de circunferência em sua região corporal de maior largura. As demais informações morfométricas e o detalhe visual de algumas áreas do corpo do animal são apresentados na Tabela 1 e Figura 5.

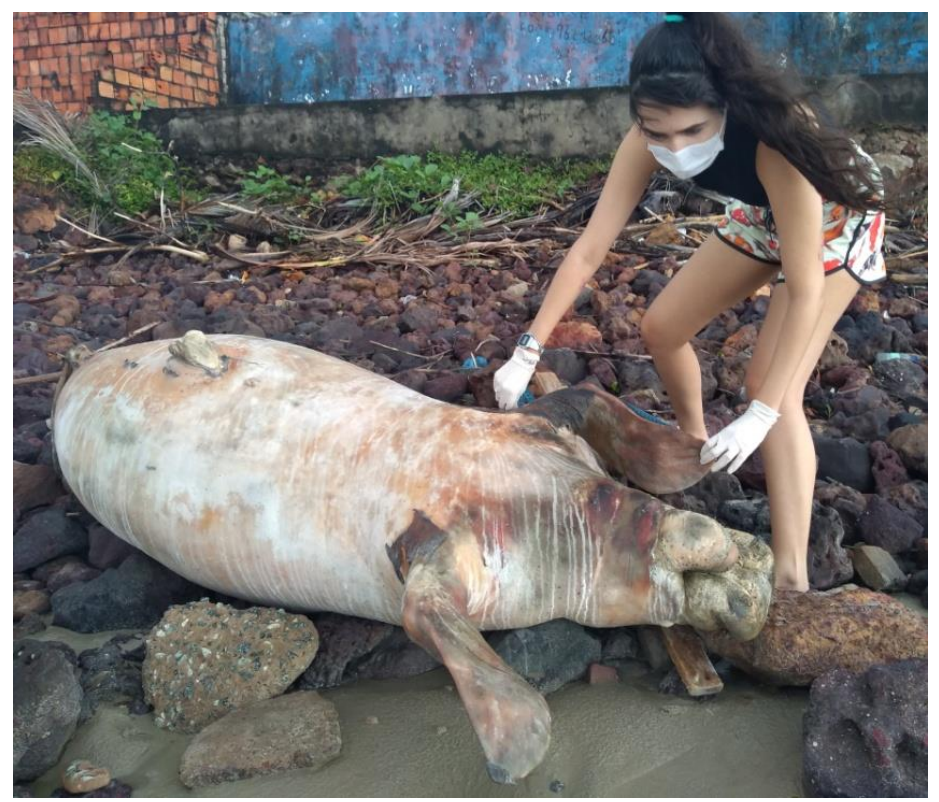

Figura 3. Procedimentos morfométricos junto ao peixe-boi-marinho-das-Antilhas encontrado na praia de Panaquatira, São José de Ribamar - MA. 

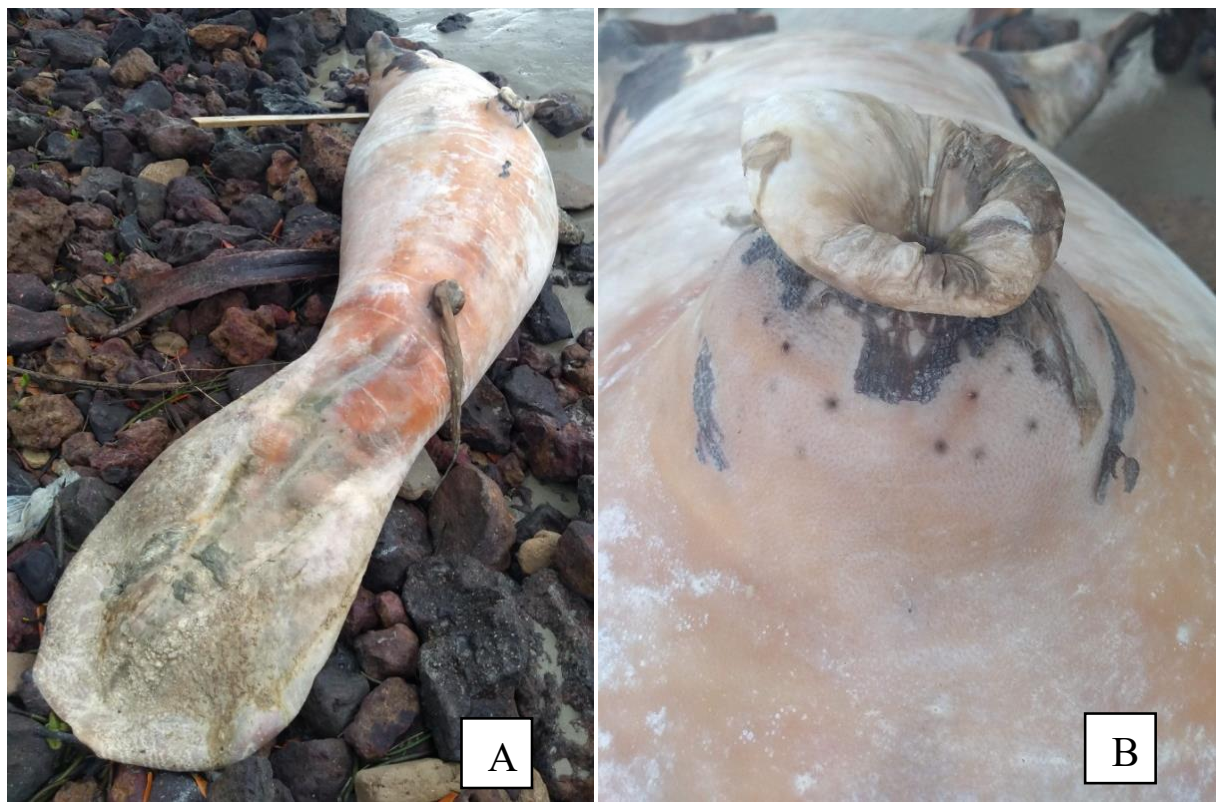

Figura 4. Vista da região ventral do peixe-boi-marinho-das-Antilhas encontrado na praia de Panaquatira, São José de Ribamar - MA, evidenciando a grande distância entre as regiões anal e genital (A) e o detalhe da abertura genital (B).

Visando caracterização e identificação genética posterior, foi realizada coleta de material biológico (tecido) oriundo da nadadeira peitoral direita. Os tecidos coletados foram depositados no Laboratório de Genética da Universidade Federal do Maranhão.

Tabela 1. Informações morfométricas do peixe-boi-marinho-das-Antilhas encontrado na praia de Panaquatira, São José de Ribamar - MA.

\begin{tabular}{lcc}
\multicolumn{1}{c}{ Parte corporal } & Largura (cm) & Comprimento (cm) \\
\hline Nadadeira caudal & 85 & 60 \\
Nadadeira peitoral direita & 50 & 20 \\
Nadadeira peitoral esquerda & 45 & 17 \\
Crânio & 20 & 63 (diâmetro) \\
\hline
\end{tabular}
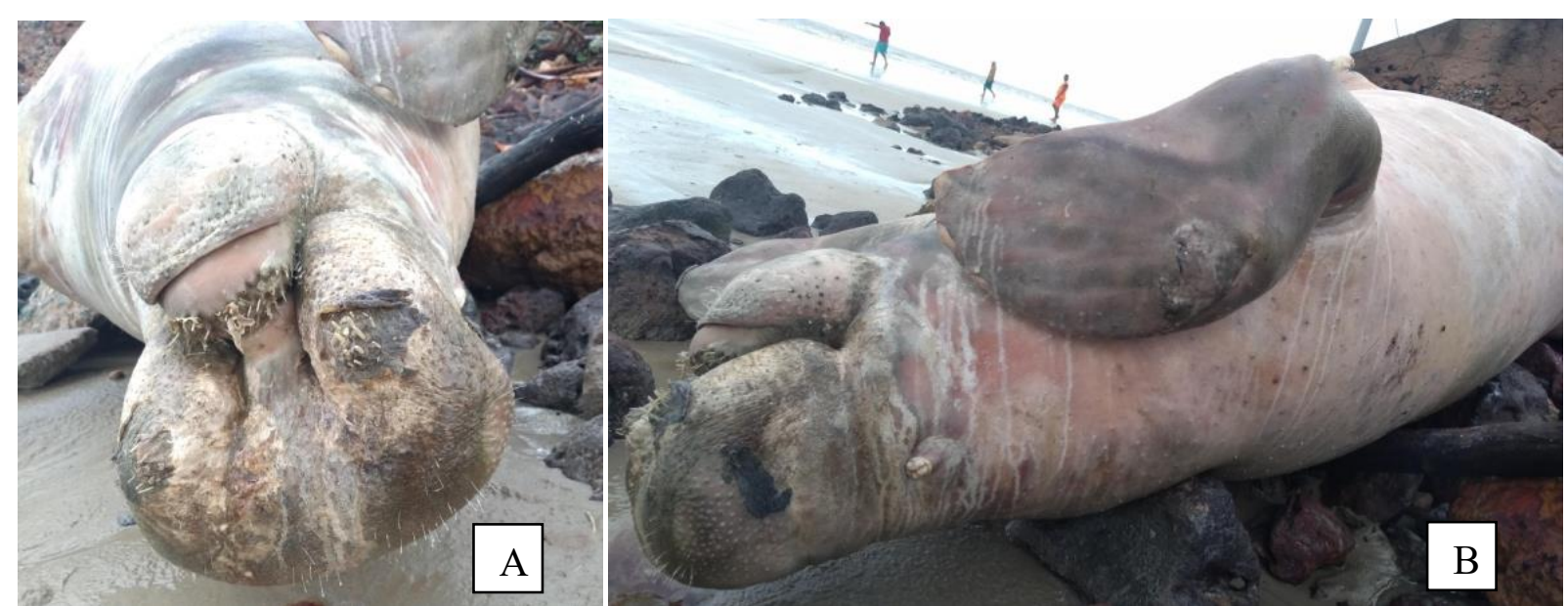

Figura 5. Vistas das regiões da cabeça (A) e lateral (B) do peixe-boi-marinho-das-Antilhas encontrado na praia de Panaquatira, São José de Ribamar - MA. 
O peixe-boi-marinho-das-Antilhas é o mamífero aquático mais ameaçado de extinção no Brasil. A ocorrência desse mamífero marinho é relatada por populações ribeirinhas nas regiões central e oeste do litoral maranhense. Porém, a pouca disponibilidade de dados científicos para essas regiões sobre o status de conservação de Trichechus m. manatus, incluindo informações sobre áreas atuais de distribuição, parâmetros populacionais e ameaças vigentes e potenciais dificultam a elaboração de ações estratégicas de conservação.

O monitoramento dos encalhes de animais vivos ou mortos, a retomada de inventários e monitoramentos que possam estimar a abundância e a densidade das populações de peixes-boi, a realização de ações de educação ambiental visando à sensibilização de diferentes atores para sua conservação e de seus ambientes, e o estímulo ao turismo embarcado não motorizado e ordenado para observação do peixe-boi marinho são fundamentais para garantir a manutenção da espécie e a geração de renda no litoral maranhense.

\section{AGRADECIMENTOS}

Os autores explicitam agradecimento à professora Larissa Barreto, da Universidade Federal do Maranhão, por suas sugestões ao presente artigo.

\section{REFERÊNCIAS BIBLIOGRÁFICAS}

Alvite, C.M.C.; Paluo, J.R. \& Lima, R.P.L. 2006. Monitoramento de peixes-bois marinhos (Trichechus manatus) no litoral do Maranhão (2003-2005). In: Resumos do VII Congresso Internacional sobre Manejo de Fauna Silvestre na Amazônia e América Latina, Ilhéus, Brasil. p. 309.

Alvite, C.M.C. 2008. Indicadores populacionais e ecológicos de peixes-bois-marinhos (Trichechus manatus manatus) em duas áreas de manguezais e marismas no Maranhão. Dissertação (Mestrado em Sustentabilidade de Ecossistemas). Centro de Ciências Biológicas e da Saúde, Universidade Federal do Maranhão, São Luís, MA. 118 p.

Best, R.C. \& Teixeira, D.M. 1982. Notas sobre a distribuição e "status" aparentes dos peixes-bois (Mammalia: Sirenia) nas costas amapaenses brasileiras. Boletim da Fundação Brasileira para a Conservação da Natureza 17: 41-47.

Borges, J.C.G.; Araújo, C.G.; Anzolin, D.G. \& Miranda, G.E.C. 2008. Identificação de itens alimentares constituintes da dieta dos peixes-boi marinhos (Trichechus manatus) na região Nordeste do Brasil. 
Biotemas 21(2): 77-81.

Domning, D.P. \& Hayek, L-A.C. 1986. Interspecific and intraspecific morphological variation in manatees (Sirenia: Trichechus). Marine Mammal Science 2: 87-144.

Hatt, R.T. 1934. A manatee collected by the American Museum Congo Expedition, with observations on the recent manatees. Bulletin of the American Museum of Natural History 66: 533-66.

Hartman, D.S. 1979. Ecology and behavior of the manatee (Trichechus manatus) in Florida. American Society of Mammalogists, Special Publication 1: 1-153.

IUCN. 2006. The 2006 IUCN Red List of Threatened Species. Disponivel em: <http://www.redlist.org>. Acesso em: 11 nov. 2006.

Luna, F.O.; Araújo, J.P.; Oliveira, E.M.; Hage, L.M. \& Passavante, J.Z.O. 2010. Distribuição do peixe-boi marinho, Trichechus manatus manatus, no litoral norte do Brasil. Arquivos de Ciências do Mar 43(2): 79 86.

Luna, F.O.; Balensiefer, D.C.; Fragoso, A.B.; Stephano, A. \& Attademo, F.L.N. 2018. Trichechus manatus Linnaeus, 1758, p.103-109. In: Instituto Chico Mendes de Conservação da Biodiversidade (Org.). Livro Vermelho da Fauna Brasileira Ameaçada de Extinção: Volume II - Mamíferos. Brasília, ICMBio. 622p.

MMA. 2014. Portaria № 444, Lista Nacional Oficial de Espécies da Fauna Ameaçadas de Extinção. Diário Oficial da União 245: 121-126.

MMA. 2018. Portaria no 249, Aprova o Plano de Ação Nacional para a Conservação do Peixe-boi Marinho. Diário Oficial da União 66: 172-175.

Paludo, D. 1997. Estudos sobre a ecologia e a conservação do peixe-boi marinho Trichechus manatus manatus no Nordeste do Brasil. Dissertação (Mestrado em Zoologia). Universidade Federal da Paraíba, João Pessoa, PB. 94 p.

Powell, J.A. 1978. Evidence of carnivory in manatees (Trichechus manatus). Journal of Mammalogy 59 (2): 442.

Rosas, F.C.W. \& Pimentel, T.L. 2001. Order Sirenia (manatees, dugongs and sea cows), p.352-362. In: Fowler, M.E. \& Cubas, Z.S. (Eds.). Biology, Medicine and Surgery of South American Wild Animals. Iowa State University Press. 536p. 